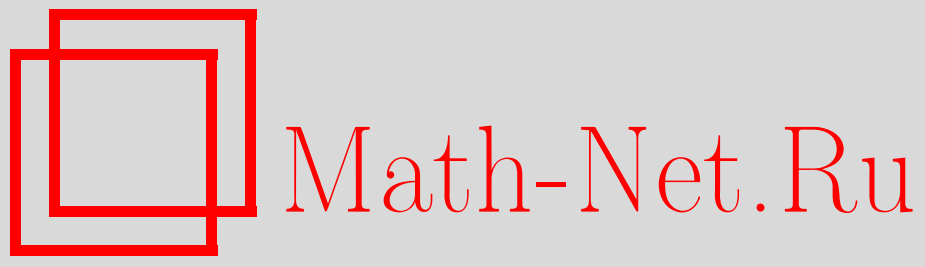

В. Я. Якубов, Оценки по спектральному параметру для собственных функций эллиптических операторов, Функи. анализ и его прил., 1999, том 33, выпуск 2, 58-67

DOI: https://doi.org/10.4213/faa354

Использование Общероссийского математического портала MathNet.Ru подразумевает, что вы прочитали и согласны с пользовательским соглашением

http://www.mathnet.ru/rus/agreement

Параметры загрузки:

IP : 54.172 .240 .79

26 апреля 2023 г., 17:41:12

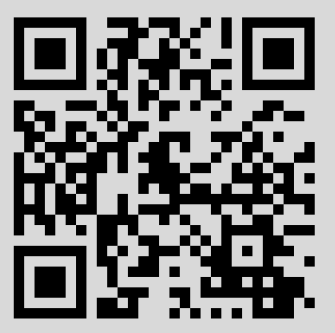


Функииональный анализ и его приложения

1999, т. 33, вып. 2, с. 58-67

УДК $517.43+517.5$

\title{
Оценки по спектральному параметру для собственных функций эллиптических операторов
}

\author{
(c) 1999. В. Я. Якуьов
}

\section{§1. Оценка сверху для собственных функций эллиптических операторов}

Получению равномерных оценок для модулей нормированных в $L_{2}$ собственных функций $u_{n}(x)$ эллиптического оператора

$$
L u \equiv \sum_{i, j=1}^{N} \frac{\partial}{\partial x_{i}}\left(a_{i j}(x) \frac{\partial u}{\partial x_{i}}\right)+a(x) u \quad(N \geqslant 2)
$$

и их производных посвящен ряд работ Х. Л. Смолицкого [1], Д. М. Эйдуса $[2,3]$, В. А. Ильина и И. А. Шишмарева [4-8], О. А. Ладыженской [9] и др.

Речь идет о нахождении наименьшего показателя $\alpha$, при котором в замкнутой области $\bar{D}=D+\Gamma$ справедлива равномерная по $x$ оценка

$$
\left|u_{n}(x)\right| \leqslant C \lambda_{n}^{\alpha} \quad(n \rightarrow+\infty),
$$

где константа $C$ зависит лишь от области $\bar{D}$ и коэффициентов оператора $L$.

В работах Ильина и Шишмарева было доказано, что в случае гладких коэффициентов $a_{i j}(x)$ в области $\bar{D}=D+\Gamma$ справедлива оценка

$$
\left|u_{n}(x)\right| \leqslant C \lambda_{n}^{(N-1) / 4} .
$$

Более того, ими было показано на примере оператора Лапласа в шаре, что эта оценка достигается. В дальнейшем эти же авторы установили, что в случае разрывных кусочно-гладких коэффициентов $a_{i j}(x)$ имеет место оценка

$$
\left|u_{n}(x)\right| \leqslant C \lambda_{n}^{N / 4} \text {. }
$$

K сожалению, вопрос о точности оценки (1.4) в классе кусочно-гладких коэффициентов остается открытым (известно, что для $n=1$ эта оценка не является точной), см., например, [19, гл. I, §2].

$\mathrm{B}$ настоящей работе показано, что если коэффициенты $a_{i j}(x)$ суммируемы в $\bar{D}$, то оценка (1.4) сохраняет силу и, более того, точна.

Отметим, что полученные нами в этом параграфе оценки стали возможны благодаря фундаментальным работам Де Джорджи, Нэша, Стампаккьи [1417] и Ладыженской.

Прежде чем формулировать теоремы, сделаем несколько замечаний.

Будем предполагать, что коэффициенты в (1.1) измеримы и, более того, удовлетворяют условиям

$$
\alpha^{-1}|\xi|^{2} \leqslant \sum_{i, j=1}^{N} a_{i j}(x) \xi_{i} \xi_{j} \leqslant \alpha|\xi|^{2},
$$


где $\alpha>0, a_{i j}=a_{j i},|a(x)| \leqslant a_{0}\left(\alpha, a_{0}\right.$ - константы). Для получения оценки (1.2) с показателем $\alpha=N / 4$ мы пользуемся теоремами 8.25 и 8.29 из [13]. Из этих теорем следует, что обобщенные решения $u(x)$ задачи Дирихле для эллиптического уравнения

$$
L u \equiv \sum_{i, j=1}^{N} \frac{\partial}{\partial x_{i}}\left(a_{i j}(x) \frac{\partial u}{\partial x_{j}}\right)+a(x) u=0,\left.\quad u\right|_{\partial D}=0
$$

с измеримыми коэффициентами, удовлетворяющими условиям (1.5), принадлежат пространству $W_{2}^{1}(D)$ и являются непрерывными функциями. Более того, для них справедлива оценка

$$
\max _{x \in \bar{D}}|u(x)| \leqslant C\|u(x)\|_{L_{2}(D)}
$$

где константа $C$ зависит лишь от чисел $\alpha, a_{0}$ и $N$. Предполагается, что граница Г области $D$ удовлетворяет условию внешнего конуса. Отметим попутно, что условия (1.5) обеспечивают существование у оператора $L u$ дискретного спектра (см. [21]).

Итак, имеет место

ТЕОРемА 1.1. Для нормированных в пространстве $L_{2}$ обобщенных собственных функций спектральной краевой задачи

$$
\sum_{i, j=1}^{N} \frac{\partial}{\partial x_{i}}\left(a_{i j}(x) \frac{\partial u}{\partial x_{j}}\right)+a(x) u+\lambda u=0,\left.\quad u\right|_{\partial D}=0
$$

с измеримьми и удовлетворяющими условиям (1.5) в области $D \subset \mathbb{R}^{N}$ (гранича области $D$ удовлетворяет условию внешнего конуса) коэффичиентами имеют место оченки

$$
\left\|u_{n}(x)\right\|_{C} \leqslant C \lambda_{n}^{N / 4}
$$

где константа $C$ зависит лишь от чисел $\alpha, a_{0} u N$.

ДоказАТЕЛьство. Пусть $x_{0}$ - точка из $\bar{D}$, такая, что $\max _{\bar{D}}|u(x)|=$ $\left|u\left(x_{0}\right)\right|$ (точка $x_{0}$ существует, так как решения задачи $(1.8)$, принадлежащие пространству $W_{2}^{1}(D)$, как было отмечено выше, непрерывны); произведем в задаче (1.8) замену

$$
t=\left(x-x_{0}\right) \lambda^{1 / 2}, \quad v(t)=u\left(x_{0}+t \lambda^{-1 / 2}\right), \quad t \in D_{1},
$$

где область $D_{1}$ есть образ области $D$. K решению $v(t)$ полученной после замены (1.10) задачи

$$
\sum_{i, j=1}^{N} \frac{\partial}{\partial t_{i}}\left(b_{i j}(t) \frac{\partial v}{\partial t_{j}}\right)+b(t) v=0,\left.\quad u\right|_{\partial D_{1}}=0
$$

применим оценку (1.7) (ясно, что коэффициенты $b_{i j}(t)$ удовлетворяют условию эллиптичности с той же константой $\alpha$, что и коэффициенты $a_{i j}(x)$, а коэффициент $b(t)$ удовлетворяет условию $\left.|b(t)| \leqslant a_{0}+1\right)$. Получаем

$$
|v(0)|=\max _{\bar{D}_{1}}|v(t)| \leqslant C\|v\|_{L_{2}\left(D_{1}\right)},
$$


где постоянная $C$ зависит лишь от констант $\alpha, a_{0}$ и размерности $N$. Но

$$
\|v\|_{L_{2}\left(D_{1}\right)}^{2}=\int_{D_{1}}|v(t)|^{2} d t=\int_{D}|u(x)|^{2} \lambda^{N / 2} d x=\lambda^{N / 2},
$$

и, следовательно, на основании (1.12) получаем, что $|v(0)| \leqslant C \lambda^{N / 4}$, или

$$
\max _{\bar{D}}|u(x)|=\left|u\left(x_{0}\right)\right|=|v(0)| \leqslant C \lambda^{N / 4},
$$

что и требовалось доказать.

\section{§ 2. Достижимость оценки (1.9)}

Основным результатом настоящей работы является доказательство достижимости оценки (1.9), и содержится он в следующей теореме.

ТЕОРема 2.1. Существует эллиптический оператор с непрерывными почти всюду в замкнутой $N$-мерной области $\bar{K}_{N}$ коэффичиентами, для счетного множества нормированных в пространстве $L_{2}$ собственньх функиий которого справедливы оченки

$$
\max _{x \in \bar{K}_{N}}\left|u_{n}(x)\right| \geqslant C \lambda_{n}^{N / 4},
$$

әде константа $C$ - число, не завислиее от $n$.

Доказательство теоремы основывается на получении соответствующего результата для собственных функций следующей обобщенной задачи ШтурмаЛиувилля:

$$
\begin{gathered}
y^{\prime \prime}+\lambda \rho(x) y=0 \quad(0<x<l), \\
y(0)=0, \quad y(l)=0, \\
\int_{0}^{l} \rho(x) y^{2}(x) d x=1,
\end{gathered}
$$

где $\rho(x)$ - измеримая на отрезке $[0, l]$ функция, удовлетворяющая условиям $0<\rho_{0} \leqslant \rho(x) \leqslant \rho_{1}$, a $l, \rho_{0}, \rho_{1}$ - заданные числа. Под собственной функцией задачи (2.1)-(2.2) мы понимаем функцию $y=y(x, \lambda, \rho) \not \equiv 0$, имеющую абсолютно непрерывную первую производную на отрезке $[0, l]$, удовлетворяющую условиям (2.2) и почти всюду уравнению (2.1).

Построим пример непрерывной почти всюду на отрезке $[0, l]$ функции $\rho(x)$, такой, что для счетного множества номеров $n$ справедливы оценки

$$
a \lambda_{n}^{1 / 4}(\rho) \leqslant \max _{0 \leqslant x \leqslant l}\left|y\left(x, \lambda_{n}(\rho), \rho\right)\right| \leqslant b \lambda_{n}^{1 / 4}(\rho),
$$

где $y\left(x, \lambda_{n}(\rho), \rho\right)$ - собственные функции задачи $(2.1)-(2.3)$, соответствующие собственным числам $\lambda_{n}(\rho)$, а числа $a>0$ и $b>0$ находятся явно и не зависят от $n$ (правое неравенство выполняется для всех $n$ ).

На отрезке $[0,3 / 4]$ введем в рассмотрение кусочно-постоянные функции

$$
\rho_{n}(x)= \begin{cases}\pi^{2}, & \text { если } x \in \bigcup_{k=1}^{n}\left[x_{2(k-1)}, x_{2 k-1}\right), \\ (9 \pi)^{2}, & \text { если } x \in \bigcup_{k=1}^{n}\left[x_{2 k-1}, x_{2 k}\right), \\ (4 \pi)^{2}, & \text { если } x \in\left[x_{2 n}, 3 / 4\right],\end{cases}
$$


где $x_{i}=(3 / 4)\left(1-1 / 3^{i}\right)(i=0,1, \ldots)$, и рассмотрим задачу $(2.1)-(2.2)$ при $\rho=\rho_{n}(x), l=3 / 4$. Собственные функции $y\left(x, \lambda_{n}(\rho), \rho_{n}\right)$ этой задачи удовлетворяют уравнению $(2.1)$ на интервалах непрерывности функции $\rho_{n}(x)$, а в точках $x_{i}(i=1, \ldots, 2 n)$ - условиям гладкой склейки: $y\left(x_{i}-0\right)=y\left(x_{i}+0\right)$, $y^{\prime}\left(x_{i}-0\right)=y^{\prime}\left(x_{i}+0\right)$. Имеет место

ЛЕмма 2.1. Функиии

$$
y\left(x, \lambda, \rho_{n}\right)= \begin{cases}3^{2(k-1)} \sin (\sqrt{\lambda} \pi x), & \text { если } x \in\left[x_{2(k-1)}, x_{2 k-1}\right], \\ 3^{2(k-1)} \sin (9 \sqrt{\lambda} \pi x), & \text { если } x \in\left[x_{2 k-1}, x_{2 k}\right], \quad k=1, \ldots, n, \\ \frac{1}{4} \cdot 3^{2 n} \sin (4 \sqrt{\lambda} \pi x), & \text { если } x \in\left[x_{2 n}, 3 / 4\right],\end{cases}
$$

являются собственньми функииями задачи (2.1)-(2.2) $\left(\rho=\rho_{n}, l=3 / 4\right)$, соответствующими собственным числам $\lambda=3^{2 m}$ при любом натуральном $m \geqslant 4 n$, причем точки $x_{2 k}$ - нули, а $x_{2 k-1}$ - абсииссь экстремумов этих

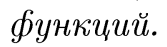

Доказательство проводится непосредственной проверкой; при этом учитывается, что числа вида $3^{2 k}-1=\left(3^{2}-1\right)\left(3^{2(k-1)}+3^{2(k-2)}+\cdots+1\right)=8 p$ кратны 4 , а числа вида $3^{2 k-1}-1=(3-1)\left(3^{2 k}+3^{2 k-1}+\cdots+1\right)=2(2 q-1)$ кратны 2 , но не кратны 4.

Обозначим теперь через $y^{*}(x, \lambda, \rho)$ решение задачи $(2.1)-(2.3)$, т. е. нормированную собственную функцию. Тогда справедлива

ЛЕмма 2.2. При любых натуральных числах $m$ u $n$, таких, что $m \geqslant 4 n$, имеют место оченки

$$
c_{1} \cdot 3^{n}<\max _{0 \leqslant x \leqslant 3 / 4}\left|y^{*}\left(x, 3^{2 m}, \rho_{n}\right)\right|<c_{2} \cdot 3^{n},
$$

где константы $c_{1}$ и $c_{2}$ ни от $n$, ни от $m$ не зависят и находятся в явном вuдe.

ДокАЗАТЕЛЬство. Найдем норму в $L_{2}$ функции $y\left(x, 3^{2 m}, \rho_{n}\right)$ :

$$
\begin{aligned}
\| y(x, & \left.3^{2 m}, \rho_{n}\right) \|_{L_{2}}^{2}=\int_{0}^{3 / 4} \rho_{n}(x) y^{2}\left(x, 3^{2 m}, \rho_{n}\right) d x \\
& =\pi^{2}\left[\sum_{k=1}^{n} 3^{4(k-1)}\left(\int_{x_{2(k-1)}}^{x_{2 k-1}} \sin ^{2}\left(3^{m} \pi x\right) d x+81 \int_{x_{2 k-1}}^{x_{2 k}} \sin ^{2}\left(3^{m+2} \pi x\right) d x\right)\right. \\
& =\frac{\pi^{2}}{2}\left\{\sum _ { k = 1 } ^ { n } 3 ^ { 4 ( k - 1 ) } \left[\left(x_{2 k-1} \int_{x_{2 n}} \sin ^{2}\left(4 \cdot 3^{m} \pi x\right) d x\right]\right.\right. \\
& =\frac{3 \pi^{2}}{8}\left\{\sum_{k=1}^{n} 3^{4(k-1)}\left[\left(\frac{1}{3^{2 k-2}}-\frac{1}{3^{2 k-1}}\right)+81\left(\frac{1}{3^{2 k-1}}-\frac{1}{3^{2 k}}\right)\right]+3^{4 n} \frac{1}{3^{2 n}}\right\} \\
& \left.=\frac{3 \pi^{2}}{8}\left(\frac{56}{27} \sum_{k=1}^{n} 3^{2 k}+3^{2 n}\right)=\frac{\pi^{2}}{4}\left(5 \cdot 3^{2 n}-\frac{7}{27}\right)=\frac{\pi^{2}}{4}\left(5-\frac{3}{4}-3^{4 n}\right)\right\}
\end{aligned}
$$


Таким образом,

$$
\left\|y\left(x, 3^{2 m}, \rho_{n}\right)\right\|_{L_{2}}=\frac{\pi}{2} \sqrt{5-\frac{7}{2 \cdot 3^{2 n}}} 3^{n} .
$$

Из (2.4) очевидным образом следует, что при $m \geqslant 4 n$

$$
\max _{0 \leqslant x \leqslant 3 / 4}\left|y\left(x, 3^{2 m}, \rho_{n}\right)\right|=\max _{x_{2 n} \leqslant x \leqslant 3 / 4}\left|y\left(x, 3^{2 m}, \rho_{n}\right)\right|=\frac{1}{4} \cdot 3^{2 n} .
$$

Из (2.6) и (2.5) теперь получаем

$\max _{0 \leqslant x \leqslant 3 / 4}\left|y^{*}\left(x, 3^{2 m}, \rho_{n}\right)\right|=\max _{0 \leqslant x \leqslant 3 / 4} \frac{\left|y\left(x, 3^{2 m}, \rho_{n}\right)\right|}{\left\|y\left(x, 3^{2 m}, \rho_{n}\right)\right\|_{L_{2}}}=\frac{1}{2 \pi}\left(5-\frac{7}{2 \cdot 3^{2 n}}\right)^{-1 / 2} \cdot 3^{n}$, откуда следует

$$
\frac{1}{2 \sqrt{5} \pi} \cdot 3^{n}<\max _{0 \leqslant x \leqslant 3 / 4}\left|y^{*}\left(x, 3^{2 m}, \rho_{n}\right)\right|<\frac{1}{4 \pi} \cdot 3^{n},
$$

что и требовалось доказать.

СлЕДСТвИЕ. Для собственной функиии $y^{*}\left(x, \lambda\left(\rho_{n}\right), \rho_{n}\right)$ задачи (2.1)-(2.3) $\left(\rho=\rho_{n}(x), l=3 / 4\right)$, соответствуюшей собственному значению $\lambda=3^{4 n}$, при любом достаточно большом $n \in \mathbb{N}$ имеют место оченки

$$
\frac{1}{2 \sqrt{5} \pi} \lambda^{1 / 4}<\max _{0 \leqslant x \leqslant 3 / 4}\left|y^{*}\left(x, \lambda, \rho_{n}\right)\right|<\frac{1}{4 \pi} \lambda^{1 / 4} .
$$

Рассмотрим теперь задачу Коши

$$
\begin{gathered}
y^{\prime \prime}+\lambda \rho(x) y=0 \quad(0<x<3 / 4), \\
y(0)=0, \quad y^{\prime}(0)=1,
\end{gathered}
$$

где $\rho(x)$ - измеримая ограниченная на отрезке $[0,3 / 4]$ функция. Под решением уравнения $(2.8)$ понимается функция $y(x, \lambda, \rho)$ с абсолютно непрерывной первой производной, которая почти всюду на $(0,3 / 4)$ удовлетворяет уравнению (2.8). Приведем еще ряд лемм.

Лемма 2.3. Пусть

$$
\begin{gathered}
a_{0}(x, \rho)=x, \\
a_{p}(x, \rho)=\int_{0}^{x} \int_{0}^{t_{1}} \rho\left(t_{2}\right) \int_{0}^{t_{2}} \int_{0}^{t_{3}} \rho\left(t_{4}\right) \cdots \int_{0}^{t_{2 p-2}} \int_{0}^{t_{2 p-1}} t_{2 p} \rho\left(t_{2 p}\right) d t_{2 p} \ldots d t_{1} ;
\end{gathered}
$$

тогда функция

$$
y(x, \lambda, \rho)=\sum_{p=0}^{\infty}(-\lambda)^{p} a_{p}(x, \rho)
$$

является решением задачи (2.8)-(2.9).

ДокАЗАТЕЛЬСтво. Прежде всего замечаем, что

$$
\left|a_{p}(x, \rho)\right| \leqslant \frac{M}{(2 p+1) !}, \quad\left|a_{p}^{\prime}(x, \rho)\right| \leqslant \frac{M}{(2 p) !}, \quad\left|a_{p}^{\prime \prime}(x, \rho)\right| \leqslant \frac{M}{(2 p-1) !},
$$

где $M=\sup _{0 \leqslant x \leqslant 3 / 4}|\rho(x)|$, причем первые два неравенства выполняются на всем отрезке $[0,3 / 4]$, а последнее - почти всюду. Следовательно, ряд (2.11) и его производные равномерно и абсолютно сходятся. Тот факт, что сумма этого ряда есть решение задачи (2.8)-(2.9), проверяется легко. 
Лемма 2.4. Для любых иисел $\lambda>0, \varepsilon>0$ существуют иисла $n_{0} \in \mathbb{N}$, $h>0$, такие, что при $\left|\lambda-\lambda_{1}\right|<h, n \geqslant n_{0}$ равномерно по $x \in[0,3 / 4]$ выполняется оченка

$$
\left|y\left(x, \lambda_{1}, r(x)\right)-y\left(x, \lambda, \rho_{n}(x)\right)\right|<\varepsilon,
$$

әде $r(x)=\lim _{n \rightarrow \infty} \rho_{n}(x)$.

Доказательство. Пусть $\tau(x)=r(x)-\rho_{n}(x)$. Тогда $\tau(x) \equiv 0$ при $x \in$ $\left[0, x_{2 n}\right]$ и $\tau(x) \leqslant 80 \pi^{2}$ при $x \in\left[x_{2 n}, 3 / 4\right]$ и, следовательно,

$$
\left|\int_{0}^{3 / 4} \tau(x) d x\right|=\left|\int_{x_{2 n}}^{3 / 4} \tau(x) d x\right| \leqslant 80 \pi^{2}\left(\frac{3}{4}-x_{2 n}\right)=\frac{20 \pi^{2}}{3^{2 n-1}}=\varepsilon_{1} .
$$

Далее,

$$
\begin{aligned}
a_{p}(x, r) & =a_{p}\left(x, \rho_{n}+\tau\right) \\
& =\int_{0}^{x} \int_{0}^{t_{1}}\left(\rho_{n}+\tau\right) \cdots \int_{0}^{t_{2 p-2}} \int_{0}^{t_{2 p-1}} t_{2 p}\left(\rho_{n}\left(t_{2 p}\right)+\tau\left(t_{2 p}\right)\right) d t_{2 p} \ldots d t_{1} \\
& =\int_{0}^{x} \int_{0}^{t_{1}} \rho\left(t_{2}\right) \cdots \int_{0}^{t_{2 p-2}} \int_{0}^{t_{2 p-1}} t_{2 p} \rho_{n}\left(t_{2 p}\right) d t_{2 p} \ldots d t_{1}+\sum_{i=1}^{N} a_{i}\left(x, \rho_{n}, \tau\right) \\
& =a_{p}\left(x, \rho_{n}\right)+\sum_{i=1}^{N} a_{i}\left(x, \rho_{n}, \tau\right),
\end{aligned}
$$

где $a_{i}\left(x, \rho_{n}, \tau\right)$ - интегралы вида $(2.10)$, в которых вместо функции $\rho(x)$ на некоторых местах стоит функция $\rho_{n}(x)$, а на других - функция $\tau(x)$, и таких интегралов получается $N=C_{p}^{1}+C_{p}^{2}+\cdots+C_{p}^{p}=2^{p}-1$.

Таким образом,

$$
a_{p}(x, r)-a_{p}\left(x, \rho_{n}\right)=\sum_{i=1}^{N} a_{i}\left(x, \rho_{n}, \tau\right) .
$$

Оценим правую часть этого равенства:

Значит,

$$
\begin{gathered}
\left|a_{i}\left(x, \rho_{n}, \tau\right)\right|<\left(81 \pi^{2}\right)^{p} \int_{0}^{3 / 4}|\tau(x)| d x<\left(81 \pi^{2}\right)^{p} \varepsilon_{1}, \\
\left|\sum_{i=1}^{N} a_{i}\left(x, \rho_{n}, \tau\right)\right|<N\left(81 \pi^{2}\right)^{p} \varepsilon_{1}=\varepsilon_{2} . \\
\left|a_{p}(x, r)-a_{p}\left(x, \rho_{n}\right)\right|<\varepsilon_{2} .
\end{gathered}
$$

Перейдем к основной оценке:

$$
\begin{aligned}
& \left|y\left(x, \lambda_{1}, r(x)\right)-y\left(x, \lambda, \rho_{n}(x)\right)=\right| \sum_{p=1}^{\infty}\left(\left(-\lambda_{1}\right)^{p} a_{p}(x, r)-(-\lambda)^{p} a_{p}\left(x, \rho_{n}\right)\right) \mid \\
& \quad=\left|\sum_{p=1}^{\infty}\left(\left(-\lambda_{1}\right)^{p} a_{p}(x, r)-(-\lambda)^{p} a_{p}(x, r)+(-\lambda)^{p} a_{p}(x, r)-(-\lambda)^{p} a_{p}\left(x, \rho_{n}\right)\right)\right| \\
& \quad \leqslant \sum_{p=1}^{\infty} \lambda^{p}\left|a_{p}(x, r)-a_{p}\left(x, \rho_{n}\right)\right|+\sum_{p=1}^{\infty}\left|\lambda^{p}-\lambda_{1}^{p}\right| a_{p}(x, r)=s_{1}+s_{2} .
\end{aligned}
$$


Оценим суммы $s_{1}$ и $s_{2}$ :

$$
s_{1}=\sum_{p=1}^{k} \lambda^{p}\left|a_{p}(x, r)-a_{p}\left(x, \rho_{n}\right)\right|+\sum_{p=k+1}^{\infty} \lambda^{p}\left|a_{p}(x, r)-a_{p}\left(x, \rho_{n}\right)\right| .
$$

Берем $k$ настолько большим, чтобы вторая сумма стала меньше $\varepsilon / 3$, а для оценки первой суммы используем неравенство (2.13). Итак $s_{1}<\sum_{p=1}^{k} \lambda^{p} \varepsilon_{2}+$ $\varepsilon / 3=2 \varepsilon / 3$, если взять $n$ настолько большим, чтобы $\varepsilon_{2} \leqslant \varepsilon\left(3 \sum_{p=1}^{k} \lambda^{p}\right)^{-1}$, $\varepsilon_{1}=\varepsilon_{2}\left(N(9 \pi)^{2 p}\right)^{-1}$.

Далее,

$$
\begin{aligned}
s_{2} & =\left|\lambda-\lambda_{1}\right| \sum_{p=1}^{\infty} \frac{\left(80 \pi^{2}\right)^{p}}{(2 p+1) !}\left(\lambda^{p-1}+\lambda^{p-2} \lambda_{1}+\cdots+\lambda \lambda_{1}^{p-2}+\lambda_{1}^{p-1}\right) \\
& <h \sum_{p=1}^{\infty} \frac{p(2 \lambda)^{(p-1)}\left(80 \pi^{2}\right)^{p}}{(2 p+1) !}=\frac{\varepsilon}{3}, \quad \text { если } h=\frac{\varepsilon}{3}\left(\sum_{p=1}^{\infty} \frac{p(2 \lambda)^{(p-1)}\left(80 \pi^{2}\right)^{p}}{(2 p+1) !}\right)^{-1} .
\end{aligned}
$$

Лемма доказана.

ЛЕмма 2.5. Для любих чисел $\varepsilon>0$ и $m \in \mathbb{N}$ существует иисло $n_{0} \in \mathbb{N}$, такое, что при $n \geqslant n_{0}$

$$
\left|\lambda_{m}(r(x))-\lambda_{m}\left(\rho_{n}(x)\right)\right|<\varepsilon,
$$

аде $\lambda_{m}(r(x))$ и $\lambda_{m}\left(\rho_{n}(x)\right)$ - это т-е собственные числа задачи (2.1)-(2.2) при $\rho=r$ и $\rho=\rho_{n}$ соответственно.

ДоказАтЕльство. Задача (2.1)-(2.2) равносильна в $L_{2}(0,3 / 4)$ спектральной задаче для интегрального уравнения

$$
y(x)=\lambda \int_{0}^{3 / 4} G(x, s) \sqrt{\rho(x) \rho(s)} y(s) d s,
$$

где $G(x, s)$ - функция Грина оператора $\left\{y^{\prime \prime}=0, y(0)=y(3 / 4)=0\right\}$. Рассмотрим в $L_{2}(0,3 / 4)$ оператор $I(\rho) \varphi=\int_{0}^{3 / 4} G(x, s) \sqrt{\rho(x) \rho(s)} \varphi(s) d s$. Тогда

$$
\begin{aligned}
& \left\|I\left(\rho_{n}(x)\right)-I(r(x))\right\|^{2} \leqslant \int_{0}^{3 / 4} \int_{0}^{3 / 4}|G(x, s)|^{2}\left|\rho_{n}(x) \rho_{n}(s)-r(x) r(s)\right| d x d s \\
& \quad \leqslant \int_{0}^{3 / 4} \int_{0}^{3 / 4}\left|\rho_{n}(x) \rho_{n}(s)-\rho_{n}(x) r(s)+\rho_{n}(x) r(s)-r(x) r(s)\right| d x d s \\
& \quad \leqslant \int_{0}^{3 / 4} \int_{0}^{3 / 4}\left|\rho_{n}(x)\left(\rho_{n}(s)-r(s)\right)+r(s)\left(\rho_{n}(x)-r(x)\right)\right| d x d s \\
& \quad \leqslant 162 \pi^{2} \int_{0}^{3 / 4}\left|\rho_{n}(x)-r(x)\right| d x<\delta_{1}
\end{aligned}
$$

(ввиду (2.12) $\delta_{1}$ можно считать меньшим любого наперед заданного числа, если выбрать соответствующим образом $n$ ). Отсюда (см. [20, с. 368]) следует (2.14).

Лемма 2.6. Для любых чисел $k \in \mathbb{N}, \varepsilon>0$ существует иисло $n_{0} \in \mathbb{N}$, такое, что при $n \geqslant n_{0}$ равномерно по $x \in[0,3 / 4]$ виполняется неравенство

$$
\left|y\left(x, \lambda_{k}\left(\rho_{n}\right), \rho_{n}(x)\right)-y\left(x, \lambda_{k}(r), r(x)\right)\right|<\varepsilon,
$$


где $y(x, \lambda, \rho)$ - решение задачи Коши (2.8)-(2.9), а $\lambda_{k}(\rho)$ есть $k$-е собственное число задачи (2.1)-(2.2).

Доказательство следует из лемм 2.4 и 2.5.

ЛЕмма 2.7. Для любого числа $\varepsilon>0$ и непрерьвной на отрезке [0,3/4] функиии $\varphi(x) \not \equiv 0$ существует число $\delta>0$, такое, что для любой непрерьвной на отрезке $[0,3 / 4]$ функиии $g(x) \not \equiv 0$, удовлетворяюшей условию $\max _{0 \leqslant x \leqslant 3 / 4}|\varphi(x)-g(x)|<\delta$, имеет место оченка

$$
\max _{0 \leqslant x \leqslant 3 / 4}|| \varphi(x)\left|\left(\int_{0}^{3 / 4}|\varphi(x)|^{2} d x\right)^{-1 / 2}-\right| g(x)\left|\left(\int_{0}^{3 / 4}|g(x)|^{2} d x\right)^{-1 / 2}\right|<\varepsilon .
$$

ДокаЗАТЕЛЬСТво. Пусть $g(x)=\varphi(x)+\tau(x), M_{1}=\max _{0 \leqslant x \leqslant 3 / 4}|\varphi(x)|, \tau_{1}=$ $\max _{0 \leqslant x \leqslant 3 / 4}|\tau(x)|, M_{2}=\|\varphi(x)\|_{L_{2}}=\left(\int_{0}^{3 / 4} \varphi^{2}(x) d x\right)^{1 / 2}$ и $\tau_{1}<M_{2} / 2$.

Тогда

$$
\frac{|\varphi(x)|}{\|\varphi(x)\|_{L_{2}}}-\frac{|g(x)|}{\|g(x)\|_{L_{2}}} \leqslant \frac{|\varphi(x)|}{M_{2}}-\frac{|\varphi(x)|-\tau_{1}}{M_{2}+\tau_{1}}=\frac{\tau_{1}\left(|\varphi(x)|-M_{2}\right)}{M_{2}\left(M_{2}+\tau_{1}\right)} \leqslant \frac{\tau_{1} M_{1}}{M_{2}^{2}}<\frac{\varepsilon}{4}
$$

если взять $\tau_{1}<\varepsilon M_{2}^{2} / 4 M_{1}=\delta$. Аналогично,

$$
\begin{aligned}
\frac{|g(x)|}{\|g(x)\|_{L_{2}}}-\frac{|\varphi(x)|}{\|\varphi(x)\|_{L_{2}}} & \leqslant \frac{|\varphi(x)|+\tau_{1}}{M_{2}-\tau_{1}}-\frac{|\varphi(x)|}{M_{2}} \\
& =\frac{\tau_{1}\left(M_{2}+|\varphi(x)|\right)}{\left(M_{2}-\tau_{1}\right) M_{2}}<\frac{2 M_{1} \tau_{1}}{0,5 M_{2}^{2}}=4 \frac{\tau_{1} M_{1}}{M_{2}^{2}}<\varepsilon .
\end{aligned}
$$

Лемма доказана.

Из лемм 2.5-2.7 следует

ЛЕмма 2.8. Для любых чисел $m \in \mathbb{N} u \varepsilon>0$ существует иисло $n_{0} \in \mathbb{N}$, такое, что при всех $n \geqslant n_{0}$ для соответствуюших решений задачи (2.1)(2.3) справедливь оченки

$$
\left|y^{*}\left(x, \lambda_{m}\left(\rho_{n}\right), \rho_{n}(x)\right)-y^{*}\left(x, \lambda_{m}(r), r(x)\right)\right|<\varepsilon .
$$

Из оценок (2.7) и (2.15) следует

ЛЕмма 2.9. Существуют почти всюду непрерывные на отрезке [0,3/4] функиии $r(x)$, такие, что для счетного множества собственньх функиий задачи (2.1)-(2.3) $(l=3 / 4, \rho=r(x))$ имеют место оченки

$$
\frac{1}{2 \sqrt{5} \pi} \lambda^{1 / 4}(r)<\max _{0 \leqslant x \leqslant 3 / 4}\left|y^{*}(x, \lambda(r), r(x))\right|<\frac{1}{4 \pi} \lambda^{1 / 4}(r) .
$$

Отметим, что функцию $r(x)$ можно сделать непрерывной всюду на полуинтервале $[0,3 / 4)$ (см. [18, 19]).

Перейдем теперь к доказательству основной теоремы настоящей работы - теоремы 2.1 .

Пусть

$$
L u \equiv \sum_{i=1}^{N} \frac{\partial}{\partial x_{i}}\left(a\left(x_{i}\right) \frac{\partial u}{\partial x_{i}}\right)
$$


где функция $a\left(x_{i}\right)$ непрерывна и положительна на отрезке $[0, b]$. Рассмотрим спектральную задачу

$$
\begin{gathered}
-L u=\lambda u \quad\left(x \in K_{N}\right), \\
\left.u\right|_{\Gamma_{N}}=0,
\end{gathered}
$$

где $\Gamma_{N}$ - поверхность $N$-мерного куба $\bar{K}_{N}=\left\{0 \leqslant x_{i} \leqslant b, i=1, \ldots, N\right\}$. Пользуясь методом Фурье, сведем задачу (2.17)-(2.18) к $N$ одинаковым задачам Штурма-Лиувилля

$$
\begin{gathered}
-\frac{d}{d t}\left(a(t) \frac{d v(t)}{d t}\right)=\mu v(t), \\
v(0)=0, \quad v(b)=0 .
\end{gathered}
$$

Известным преобразованием

$$
x=\int_{0}^{t} \frac{d s}{a(s)}, \quad v(t(x))=y(x),
$$

где $t=t(x)$ - функция, обратная к функции $x=\int_{0}^{t}[a(s)]^{-1} d s$, приведем задачу (2.19)-(2.20) к задаче

$$
\begin{gathered}
y^{\prime \prime}+\mu \rho(x) y=0 \quad(0<x<l), \\
y(0)=0, \quad y(l)=0,
\end{gathered}
$$

где $\rho(x)=a(t(x)), l=\int_{0}^{b}[a(s)]^{-1} d s$.

На основании леммы 2.9 сушествует функция $\rho(x)$, непрерывная почти всюду на отрезке $[0,3 / 4]$, такая, что для счетного множества нормированных собственных функций $y_{n}(x, \rho)$ задачи $(2.21)-(2.22)$, соответствующих собственным значениям $\mu_{n}$, имеют место оценки

$$
\frac{1}{2 \sqrt{5} \pi} \mu_{n}^{1 / 4} \leqslant\left\|y_{n}(x, \rho)\right\|_{C[0,3 / 4]} .
$$

Но числа $\lambda_{n}=N \mu_{n}$ являются собственными значениями задачи (2.17)-(2.18), а соответствующие им собственные функции имеют вид

$$
u_{n}(x)=u_{n}\left(x_{1}, \ldots, x_{N}\right)=v_{n}\left(x_{1}\right) \cdots v_{n}\left(x_{N}\right) ;
$$

поэтому теорему 2.1 можно считать доказанной.

Построены также примеры эллиптических операторов, для нормированных собственных функций которых имеют место другие порядки роста [10-12].

В заключение автор выражает благодарность профессорам В. В. Жикову, В. Б. Лидскому и И. А. Шишмареву за внимание к работе.

\section{ЛитературА}

1. Смолиикий X. Л. Оценки производных фундаментальных функций. ДАН CCCP, 74, № 2, 205-208 (1950).

2. Эйдус Д. М. Оценки модуля собственных функций. ДАН СССР, 90, № 6, 973-974 (1953).

3. Эйдус Д. М. Некоторые неравенства для собственных функций, ДАН СССР, 107, № 6, 796-798 (1956). 
4. Ильин В. А., Шишмарев И. А. О точных оценках собственных функций в замкнутой области. Материалы к совместному советско-американскому симпозиуму по уравнениям в частных производных, Новосибирск, август 1963.

5. Ильин В. А., Шишмарев И. А. Равномерные в замкнутой области оценки для собственных функций эллиптического оператора и их производных. Изв. АН СССР, сер. матем., 24, №6, 883-896 (1960).

6. Шишмарев И. А. Введение в теорию эллиптических уравнений. Изд-во МГУ, 1979.

7. Шиимарев И. А. Равномерные оценки производных решений задачи Дирихле и задачи на собственные функции для оператора $L u=\operatorname{div}(p(x) \operatorname{grad} u)+q(x) u$ с разрывными коэффициентами. ДАН СССР, 137, № 1, 45-47 (1961).

8. Ильин B. A. О системе классических собственных функций линейного самосопряженного оператора с разрывными коэффициентами. ДАН СССР, 137, № 2, $272-275$ (1961).

9. Ладыженская О. А. Метод Фурье для гиперболических уравнений. ДАН СССР, 74, № 3, 417-420 (1950).

10. Якубов $B$. Я. Оценки для нормированных в $L_{2}$ собственных функций эллиптического оператора. ДАН СССР, 274, № 1, 35-37 (1984).

11. Якубов B. Я. Оценки для собственных функций эллиптического оператора с суммируемым коэффициентом. Тезисы Второй Северо-Кавказской региональной конференции «Функционально-дифференциальные уравнения и их приложения», Махачкала, 1989.

12. Якубов B. Я. Точные оценки для нормированных в $L_{2}$ собственных функций эллиптического оператора. Докл. РАН, 331, № 3, 286-287 (1993).

13. Гилбарг Д., Трудингер Н. Эллиптические дифференциальные уравнения с частными производными второго порядка. Наука, М., 1989.

14. Nash J. Continuity of solutions of parabolic and elliptic equations. Amer. J. Math., 80, o. 4, 931-954 (1958) [Имеется русский перевод: Нәш Дж. О непрерывности решений параболических и эллиптических уравнений. Математика, 4, № 1, 31-52 (1960)].

15. De Giorgi E. Sulla differenziabilita e l'analiticitâ delle estremali degli integrali multipli regolari. Memorie delle Acc. Sci. Torino, Ser. 3, t. 3, p. 1, 25-43 (1957) [Имеется русский перевод: Де Джорджи Э. О дифференцируемости и аналитичности экстремалей кратных регулярных интегралов. Математика, 4, № 6, 23-38 (1960)].

16. Stampacchia $G$. Problemi al contorno elliptici con dati discontinui dotati di soluzioni Hölderiane. Ann. Mat., 51, 1-38 (1960).

17. Киндерлерер Д., Стампаккья Г. Введение в вариационные неравенства и их приложения. Мир, М., 1983.

18. Якубов В. Я. Неклассические двусторонние точные оценки для нормированных собственных функций задачи Штурма-Лиувилля. Вестник МГУ, сер. 1, матем., механика, № 4, 37-44 (1993).

19. Якубов В. Я. Спектральные краевые задачи для дифференциальных операторов с негладкими коэффициентами. Докторская диссертация, МГУ, 1996.

20. Гохберг И. Ц., Крейн М. Г. Теория вольтерровых операторов в гильбертовом пространстве и ее приложения. Наука, М., 1967.

21. Ладыженская O. А., Уральцева $H . H$. Линейные и квазилинейные уравнения эллиптического типа. Наука, М., 1964. 